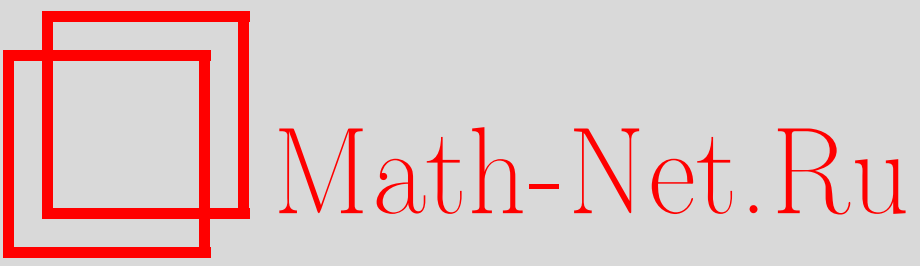

Ю. С. Вернов, М. Н. Мнацаканова, Свойства алгебры q-деформированных коммутаторов в пространстве с индефинитной метрикой, ТМФ, 1997, том 113, номер 3, 355-368

DOI: https://doi.org/10.4213/tmf1085

Использование Общероссийского математического портала Math-Net.Ru подразумевает, что вы прочитали и согласны с пользовательским соглашением

http://www. mathnet.ru/rus/agreement

Параметры загрузки:

IP: 52.6 .47 .48

26 апреля 2023 г., 03:24:16 


\title{
СВОЙСТВА АЛГЕБРЫ $q$-ДЕФОРМИРОВАННЫХ КОММУТАТОРОВ В ПРОСТРАНСТВЕ С ИНДЕФИНИТНОЙ МЕТРИКОЙ
}

\author{
В пространстве с индефинитной метрикой исследуются представления, инвариант-
} ные свойства и обобщение алгебры $q$-коммутаторов.

\section{1. ВВЕДЕНИЕ}

Алгебра $q$-деформированных коммутаторов (для краткости $q$-алгебра) есть по определению алгебра операторов $a, a^{+}$и $N$, удовлетворяюших следуюшим соотношениям:

$$
\begin{aligned}
{\left[a, a^{+}\right]_{q} } & =q^{-N}, & {\left[a, a^{+}\right]_{q} } & \equiv a a^{+}-q a^{+} a, \\
{[N, a] } & =-a, & {\left[N, a^{+}\right] } & =a^{+} .
\end{aligned}
$$

Мы предполагаем, что операторы $a$ и $a^{+}$сопряжены относительно скалярного произведения $\langle\cdot, \cdot\rangle$, которое в обшем случае является индефинитным. Оператор $N$ самосопряженный, $q \in \mathbb{R}$ или $q \in \mathbb{C},|q|=1$. Эта алгебра связана с квантовыми группами, свойства которых интенсивно исследовались в последние годы [1-3]. $q$-Алгебра в гильбертовом пространстве рассматривалась во многих статьях [4-8]. Свойства этой алгебры в пространстве с индефинитной метрикой изучались в $[9,10]$. Предлагаемая статья является продолжением этих работ.

Отметим, что, с одной стороны, $q$-алгебра находит применение в теории поля [11], а с другой стороны, пространства с индефинитной метрикой широко используются в калибровочной квантовой теории поля [12-14].

Статья построена следуюшим образом. Сначала (разделы 2,3 ) рассматриваются алгебры более обшего вида, чем $q$-алгебра, а именно алгебры, в которых условие (1) заменено условием

$$
a^{+} a=\varphi(N), \quad a a^{+}=\varphi_{1}(N),
$$

где $\varphi(N)$ и $\varphi_{1}(N)$ - некоторые функции. Нами исследуются представления, в которых эти функции несингулярны. Доказывается, что в случае, когда оператор $N$ имеет собственный вектор

$$
N \psi_{\lambda}=\lambda \psi_{\lambda}
$$

* Институт ядерных исследований РАН. E-mail: vernov@theory.npi.msu.su

${ }^{\dagger}$ Научно-исследовательский институт ядерной физики МГУ.E-mail:mnatsak@theory.npi.msu.su 
а представление рассматриваемой алгебры неприводимо, спектр $N$ может быть построен единым образом для всех алгебр указанного типа и состоит из чисел $\lambda+k$, где $k \in \mathbf{Z}$. Подчеркнем, что такой тип спектра обычно рассматривается в случае положительной метрики и предполагается, что только в этом случае соответствуюшие представления интересны для квантовой теории.

Далее изучены характерные свойства пространств, соответствуюших неприводимым представлениям указанных алгебр, и для этих алгебр получен аналог теоремы единственности фон Неймана [15].

Вторая часть работы посвяшена свойствам $q$-алгебры. В разделе 4 получено общее соотношение между операторами $a^{+} a$ и $N$ (формулы $(26)$ и $\left(26^{\prime}\right)$ ). Это соотношение содержит произвольную действительную константу $c$, в связи с чем при заданном операторе $N$ существует цельй набор операторов $a$ и $a^{+}$, вместе с $N$ образующих $q$-алгебру. В каждом конкретном представлении $q$-алгебры константа $c$ фиксирована и формулы (26) и $\left(26^{\prime}\right)$ являются частным случаем формул (3). Для гильбертова пространства аналогичная формула получена в [5]. В том же разделе рассмотрена зависимость спектра оператора $N$ от величины $c$. В частности, показано, что при “почти всех" положительных $c$ спектр $\operatorname{Sp} N$ неограничен, а метрика соответствуюшего пространства индефинитна.

В разделах 5, 6 изучены неунитарные преобразования, оставляющие $q$-алгебру инвариантной. Доказано, что если в некотором пространстве $J$ может быть реализовано какое-либо неприводимое представление $q$-алгебры, то в случае, когда $q>0, c>0$, в том же пространстве сушествуют представления с произвольными положительными константами $c$ при условии, что тип спектра соответствующего оператора $N$ не меняется (т.е. при переходе к новому представлению спектр остается неограниченным или ограниченным снизу (сверху)). Такого же типа результаты получены для представлений, в которых $q>0, c<0$, а также для представлений с $q<0$.

В разделе 7 рассмотрена связь между представлениями $q$-алгебр при различных $q$. Особенно интересным оказывается случай $q>0, c>0$. При этих условиях из сушествования в пространстве $J$ представлений $q$-алгебры с некоторыми фиксированными $q>0$, $c>0$ вытекает сушествование (в том же пространстве) представлений $q$-алгебры при произвольных положительных $q$ и $c$ при условии, что тип спектра соответствующего оператора $N$ не меняется.

В разделе 8 рассмотрено разложение представлений рассматриваемых алгебр на неприводимые представления.

В приложении обсуждаются свойства представлений $q$-алгебры при комплексных $q$. Показано, что отказ от условия положительной определенности метрики приводит к сушественному расширению набора представлений $q$-алгебры.

\section{2. СВОЙСТВА ПРОСТРАНСТВА НЕПРИВОДИМОГО ПРЕДСТАВЛЕНИЯ РАССМАТРИВАЕМЫХ АЛГЕБР}

Пусть $J$ - линейное пространство, в котором реализовано неприводимое представление алгебры, заданной условиями (2) и (3). Для произвольных векторов этого пространства определено индефинитное скалярное произведение, относительно которого операторы $a$ и $a^{+}$сопряжены, а оператор $N$ самосопряжен. Как будет показано ниже, условие сушествования собственного вектора у оператора $N$ приводит к тому, что $J$ оказывает- 
ся натянутым на собственные векторы оператора $N$, т.е. произвольный вектор этого пространства есть линейная комбинация собственных векторов оператора $N$. При этом $J$ содержит все конечные линейные комбинации этих векторов. Мы предполагаем, что соответствуюшее пространство $J_{0}$ слабо плотно в $J$, т.е. что $\forall x, y \in J$ :

$$
\langle x, y\rangle=\lim _{n \rightarrow \infty}\left\langle x_{n}, y_{n}\right\rangle, \quad x_{n}, y_{n} \in J_{0}
$$

Поскольку, как это будет видно ниже, в обшем случае $a, a^{+}$и $N$ - неограниченные операторы, необходимо сделать дополнительные утверждения об областях определения этих операторов. Мы предполагаем, что сушествует плотная в $J$ область $D: D=D\left(a^{+} a\right) \cap$ $D\left(a a^{+}\right) \cap D(N)$ и что соотношения (2) и (3) выполнены в этой области. Условия (2) эквивалентны равенствам

$$
N a=a(N-1), \quad N a^{+}=a^{+}(N+1) .
$$

Точный смысл равенств $\left(2^{\prime}\right)$ состоит в том, что если для какого-то вектора $\psi$ определено действие оператора $a(N-1) \quad\left(a^{+}(N+1)\right)$, то определено и действие оператора $N a$ $\left(N a^{+}\right)$, и наоборот, причем выполнены равенства $\left(2^{\prime}\right)$. По определению $D \subset D(a)$ и $D \subset D\left(a^{+}\right)$.

Мы рассматриваем представления, в которых оператор $N$ имеет собственный вектор (см. (4)). Согласно условиям (3) $\psi_{\lambda} \in D$. Далее, поскольку $D \subset D(a)$ и $D \subset D\left(a^{+}\right)$, то сушествуют векторы $a \psi_{\lambda}$ и $a^{+} \psi_{\lambda}$. В силу соотношений $\left(2^{\prime}\right)$ они являются собственными векторами оператора $N$, т.е.

$$
\begin{aligned}
N a^{+} \psi_{\lambda} & =(\lambda+1) a^{+} \psi_{\lambda}, \\
N a \psi_{\lambda} & =(\lambda-1) a \psi_{\lambda} .
\end{aligned}
$$

Продолжая этот процесс, мы видим, что векторы $\psi_{\lambda+n}=\left(a^{+}\right)^{n} \psi_{\lambda}$ и $\psi_{\lambda-n}=a^{n} \psi_{\lambda}$ являются собственными векторами $N$ :

$$
N \psi_{\lambda+k}=(\lambda+k) \psi_{\lambda+k},
$$

где $k$ здесь и далее - целое число.

Легко видеть,что векторы $\psi_{\lambda+k}$ исчерпывают множество собственных векторов оператора $N$. Действительно, согласно (3) и (6)

$$
\begin{aligned}
a^{+} \psi_{\lambda-n} & =a^{+} a \psi_{\lambda-n+1}=\varphi(\lambda-n+1) \psi_{\lambda-n+1}, \\
a \psi_{\lambda+n} & =a a^{+} \psi_{\lambda+n-1}=\varphi_{1}(\lambda+n-1) \psi_{\lambda+n-1} .
\end{aligned}
$$

Из формул (7) и (8) заключаем, что

$$
M\left(a^{n}, a^{+m}\right) \psi_{\lambda} \sim \psi_{\lambda+m-n}
$$

где $M\left(a^{n}, a^{+m}\right)$ - произвольный моном $n$ операторов $a$ и $m$ операторов $a^{+}$. Следовательно,

$$
P_{n}\left(a, a^{+}\right) \psi_{\lambda}=\sum_{k} c_{k} \psi_{\lambda+k}
$$


где $P_{n}\left(a, a^{+}\right)$- произвольный полином от $a$ и $a^{+}$.

Рассмотрим $J_{0}$-пространство всех векторов вида $P_{n}\left(a, a^{+}\right) \psi_{\lambda}$, т.е. согласно (9) пространство всех конечных линейных комбинаций векторов $\psi_{\lambda+k}$. Очевидно, что $D \supset J_{0}$.

Легко убедиться в том, что $J_{0}$ не содержит подпространств, инвариантных относительно рассматриваемой алгебры. Таким образом, полученное представление неприводимо. Легко также проверить, что любой $x \in J_{0}$ является циклическим вектором, как и должно быть для неприводимого представления (см. [16, с. 302]).

ЗАмЕЧАнИЕ. Если оператор $N$ имеет простой дискретный спектр, то соотношения (3) являются следствием формул (2).

Действительно, согласно $\left(2^{\prime}\right)\left[a^{+} a, N\right]=\left[a a^{+}, N\right]=0$ и $a^{+} a \psi_{\lambda+k} \sim a a^{+} \psi_{\lambda+k} \sim$ $\psi_{\lambda+k}$ вследствие простоты спектра оператора $N$. Поскольку пространство $J_{0}$ натянуто на векторы $\psi_{\lambda+k}$, то $a^{+} a=\varphi(N), a a^{+}=\varphi_{1}(N)$. Сделанное утверждение доказано.

Пространство $J$, вообще говоря, не обязано быть полным пространством. Однако, введя на $J$ гильбертову метрику (строго говоря, это можно сделать разными способами), мы можем пополнить $J$ и построить замкнутое пространство $J^{\prime}$. Поскольку рассматриваемое представление неприводимо, то $J^{\prime}=\bar{J}_{0}$, где $\bar{J}_{0}$ - замыкание пространства $J_{0}$ (относительно введенной метрики). Различные (т.е. содержашие разные бесконечные последовательности векторов $\left.\psi_{\lambda+k}\right)$ пространства $J$ содержат $J_{0}$ в качестве слабо плотной области. Уместно не различать представления, совпадающие на $J_{0}$, и считать, что $J=\bar{J}_{0}$.

Существует естественный способ построения $\bar{J}_{0}$, который в случае положительной метрики совпадает с обычной процедурой пополнения предгильбертова пространства. Сначала перейдем к системе ортонормированных векторов $\tilde{\psi}_{\lambda+k}$ :

$$
\tilde{\psi}_{\lambda+k}=\frac{\psi_{\lambda+k}}{\left|\left\langle\psi_{\lambda+k}, \psi_{\lambda+k}\right\rangle\right|^{1 / 2}} .
$$

Так как $N=N^{+}$, то

$$
\left\langle\tilde{\psi}_{\lambda+k}, \tilde{\psi}_{\lambda+l}\right\rangle=\delta_{k l} \delta_{k}, \quad \delta_{k}= \pm 1
$$

Формула (11) подразумевает, что множество $\psi_{\lambda+k}$ не содержит нейтральных векторов, т.е. что $\left\langle\psi_{\lambda+k}, \psi_{\lambda+k}\right\rangle \neq 0 \forall k$. Это условие фактически не является дополнительным предположением, т.к. согласно полученным ниже формулам $(17),(18)$ и приведеннным там же рассуждениям в этом случае либо $J_{0}$ - изотропное пространство, т.е. $\langle x, y\rangle=0 \forall x, y \in J_{0}$, либо рассматриваемое представление не является неприводимым. Мы исключаем возможность того, что $J_{0}$ - изотропное пространство, поскольку рассматриваемые задачи в таком пространстве не представляют физического интереса.

Разбив все множество векторов $\tilde{\psi}_{\lambda+k}$ на множество положительных $\tilde{\psi}_{\lambda+k}^{+}$и отрицательных $\tilde{\psi}_{\lambda+k}^{-}$векторов, мы видим, что $J_{0}$ допускает фундаментальное разложение (cм. $[17$, c. 24])

$$
J_{0}=J_{0}^{+} \oplus J_{0}^{-},
$$

где $J_{0}^{+}\left(J_{0}^{-}\right)$- пространство всех конечных линейных комбинаций векторов $\tilde{\psi}_{\lambda+k}^{+}$ $\left(\tilde{\psi}_{\lambda+k}^{-}\right)$.

Векторы

$$
x^{ \pm}=\sum_{-\infty}^{\infty} c_{k}^{ \pm} \tilde{\psi}_{\lambda+k}^{ \pm}
$$


могут принадлежать $J$, только если

$$
\left|\left\langle x^{ \pm}, x^{ \pm}\right\rangle\right|<\infty
$$

т.е. если

$$
\sum_{-\infty}^{\infty}\left|c_{k}^{ \pm}\right|^{2}<\infty .
$$

Предполагая, что последнее условие является не только необходимым, но и достаточным, для того чтобы $x^{ \pm} \in \bar{J}_{0}^{ \pm}$(иными словами, используя для пополнения $J_{0}^{ \pm}$исходное скалярное произведение $\langle\cdot, \cdot\rangle)$, построим $\bar{J}_{0}$ в виде

$$
\bar{J}_{0}=\bar{J}_{0}^{+} \oplus \bar{J}_{0}^{-} .
$$

$\mathrm{K}$ тому же пространству $\bar{J}_{0}$ можно прийти, введя на $J_{0}$ гильбертову метрику следующим способом. Поскольку для всех $x \in J_{0}$ справедливо разложение

$$
x=x^{+}+x^{-}, \quad x^{ \pm} \in J_{0}^{ \pm},
$$

то легко проверить, что $(x, y) \equiv\left\langle x^{+}, y^{+}\right\rangle-\left\langle x^{-}, y^{-}\right\rangle$- положительно-определенное скалярное произведение. Замыкая $J_{0}$, используя топологию, заданную $(x, x)$, приходим к пространству $\bar{J}_{0}$. Таким образом, мы реализовали $\bar{J}_{0}$ как пространство всех векторов вида

$$
x=\sum_{k=-\infty}^{k=+\infty} c_{k} \tilde{\psi}_{\lambda+k},
$$

для которых выполнено условие

$$
\sum_{k=-\infty}^{k=+\infty}\left|c_{k}\right|^{2}<\infty .
$$

Согласно (13) $\bar{J}_{0}$ является пространством Крейна [17, с. $100 ; 18$, с. 26].

Докажем теперь, что скалярные произведения в $J$ однозначно определяются числом $\lambda$ и видом функции $\varphi(N)$. Сначала покажем, что $\varphi_{1}(N)=\varphi(N+1)$. Действительно,

$$
\begin{aligned}
a^{+} a a^{+} \psi_{\lambda+n} & =\varphi(\lambda+n+1) \psi_{\lambda+n+1}=\varphi_{1}(\lambda+n) \psi_{\lambda+n+1}, \\
a a^{+} a \psi_{\lambda-n} & =\varphi(\lambda-n) \psi_{\lambda-n-1}=\varphi_{1}(\lambda-n-1) \psi_{\lambda-n-1},
\end{aligned}
$$

далее,

$$
\begin{aligned}
& \left\langle\psi_{\lambda+n}, \psi_{\lambda+n}\right\rangle=\left\langle\psi_{\lambda+n-1}, a a^{+} \psi_{\lambda+n-1}\right\rangle=\varphi(\lambda+n)\left\langle\psi_{\lambda+n-1}, \psi_{\lambda+n-1}\right\rangle, \\
& \left\langle\psi_{\lambda-n}, \psi_{\lambda-n}\right\rangle=\left\langle\psi_{\lambda-n+1}, a^{+} a \psi_{\lambda-n+1}\right\rangle=\varphi(\lambda-n+1)\left\langle\psi_{\lambda-n+1}, \psi_{\lambda-n+1}\right\rangle .
\end{aligned}
$$

Из соотношений (15) и (16) следует, что

$$
\begin{aligned}
\left\langle\psi_{\lambda+n}, \psi_{\lambda+n}\right\rangle & =\varphi(\lambda+n) \varphi(\lambda+n-1) \ldots \varphi(\lambda+1)\left\langle\psi_{\lambda}, \psi_{\lambda}\right\rangle, \\
\left\langle\psi_{\lambda-n}, \psi_{\lambda-n}\right\rangle & =\varphi(\lambda-n+1) \varphi(\lambda-n+2) \ldots \varphi(\lambda)\left\langle\psi_{\lambda}, \psi_{\lambda}\right\rangle .
\end{aligned}
$$


Отметим, что если $\varphi(\lambda+m)=0$ при каком-то $m \in \mathbf{Z}^{+}$, то это означает, что спектр $N$ ограничен сверху, т.е. что $\psi_{\lambda+m}=0$. Действительно, $\left\langle\psi_{\lambda+m}, \psi_{\lambda+m}\right\rangle=0$ и тогда $\left\langle\psi_{\lambda+m+n}, \psi_{\lambda+m+n}\right\rangle=0 \forall n \in \mathbf{Z}^{+}$. Легко видеть, что пространство $D_{0}$, образованное векторами вида $P_{n}\left(a, a^{+}\right) \psi_{\lambda+m}$, ортогонально $J_{0}$ и инвариантно относительно рассматриваемой алгебры. Действительно,

$$
\left\langle P_{n}\left(a, a^{+}\right) \psi_{\lambda+m}, P_{l}\left(a, a^{+}\right) \psi_{\lambda}\right\rangle=\left\langle\psi_{\lambda+m}, P_{n}^{+}\left(a, a^{+}\right) P_{l}\left(a, a^{+}\right) \psi_{\lambda}\right\rangle=0 .
$$

Если $\varphi(\lambda-n) \neq 0 \forall n$, то

$$
P_{l}\left(a, a^{+}\right) \psi_{\lambda+m}=\sum_{n} c_{n} \psi_{\lambda+m+n}
$$

Последнее равенство противоречит неприводимости рассматриваемого представления.

Аналогичные рассуждения приводят к тому, что если $\varphi(\lambda-m)=0$, а $\varphi(\lambda+n) \neq 0$ $\forall n$, то $\psi_{\lambda-m-1}=0$ и спектр $N$ ограничен снизу; а также, если $\varphi\left(\lambda+m_{1}\right)=0$ и $\varphi(\lambda-$ $\left.m_{2}\right)=0$, то спектр $N$ состоит из чисел $\lambda+k$, где $-m_{2} \leq k<m_{1}$.

Легко убедиться в том, что пространство $J_{0}$, а следовательно, и $J$ не вырождены, т.е. $x=0$, если $x \perp J_{0}$.

\section{3. АНАЛОГ ТЕОРЕМЫ ФОН НЕЙМАНА \\ ДЛЯ РАССМАТРИВАЕМЫХ АЛГЕБР}

Согласно теореме фон Неймана [15], два неприводимых представления канонических коммутационных соотношений (KKC) в гильбертовом пространстве

$$
b_{i} b_{i}^{+}-b_{i}^{+} b_{i}=1, \quad i=1,2,
$$

связаны между собой унитарным преобразованием, если только эти представления удовлетворяют некоторым "естественным" условиям. Аналог этой теоремы для КKС в пространстве с индефинитной метрикой получен в [19], а для $q$-алгебры - в [9].

Здесь мы докажем, что и в случае алгебр, заданных соотношениями (3), при определенных условиях неприводимые представления этих алгебр оказываются унитарно-эквивалентными. Ниже, вплоть до раздела 8 , мы рассматриваем только неприводимые представления и не оговариваем этого.

Tеорема. Пусть $R_{i} \quad(i=1,2)$ - представления алгебры операторов $a_{i}, a_{i}^{+}$, $N_{i}$ в пространствах $J_{i}$ - определены условиями (2) и (3). Eсли $\varphi_{2}\left(\lambda_{2}+k\right)=$ $\varphi_{1}\left(\lambda_{1}+k\right), \forall k$ (см. формулы $\left.(17),(18)\right)$, то операторы $a_{2}$ и $a_{1}$ связаны следующими преобразованиями:

1) унитарныцм -

$$
a_{2}=V a_{1} V^{+}, \quad V V^{+}=V^{+} V=I,
$$

$е с л u\left\langle\psi_{\lambda_{1}}^{(1)}, \psi_{\lambda_{1}}^{(1)}\right\rangle=\left\langle\psi_{\lambda_{2}}^{(2)}, \psi_{\lambda_{2}}^{(2)}\right\rangle$,

2) квазиунитарньм -

$$
a_{2}=-V a_{1} V^{+}, \quad V V^{+}=V^{+} V=-I,
$$


$e c \bumpeq u\left\langle\psi_{\lambda_{1}}^{(1)}, \psi_{\lambda_{1}}^{(1)}\right\rangle=-\left\langle\psi_{\lambda_{2}}^{(2)}, \psi_{\lambda_{2}}^{(2)}\right\rangle$.

Достаточно доказать соотношение (20), т.к. формула (21) вьводится аналогично.

Пусть $\psi_{\lambda_{2}+k}^{(2)}=V \psi_{\lambda_{1}+k}^{(1)}$. Соответствие между $J_{1}$ и $J_{2}$ взаимно однозначное, т.к. $x_{2}=V x_{1}$, если $x_{i}=\sum c_{k} \psi_{\lambda_{i}+k}^{i}$. Поскольку $\varphi_{2}\left(\lambda_{2}+k\right)=\varphi_{1}\left(\lambda_{1}+k\right)$, то согласно формулам (17) и (18) $\left\langle x_{2}, y_{2}\right\rangle=\left\langle x_{1}, y_{1}\right\rangle$, если $x_{2}=V x_{1}, y_{2}=V y_{1}$. Поскольку $x_{1}$ и $y_{1}-$ произвольные векторы, а пространства $J_{1}$ и $J_{2}$ не вырождены, это равенство означает, что $V^{+} V=I$. Кроме того, и $V V^{+}=I$, т.к. оператор $V^{-1}$ сушествует.

Таким образом, мы видим, что если $\varphi_{2}\left(N_{2}\right)=\varphi_{1}\left(N_{1}\right)$ и $\operatorname{Sp} N_{1}=\operatorname{Sp} N_{2}$, то представления $R_{1}$ и $R_{2}$ (квази-)унитарно-эквивалентны; (квази-)унитарные преобразования не исчерпывают всех преобразований, относительно которых $q$-алгебра инвариантна. Неунитарные преобразования рассмотрены ниже (разделы 5,6 ).

\section{4. СООТНОШЕНИЕ МЕЖДУ ОПЕРАТОРАМИ $a^{+} a$ И $N$ В СЛУЧАЕ $q$-АЛГЕБРЫ}

Найдем вид функции $\varphi(N)$ в случае $q$-алгебры. Запишем $\varphi(N)$ в виде

$$
\varphi(N)=\varphi_{1}(N)+\varphi_{2}(N) .
$$

Если $\varphi_{1}(N)$ выбрана таким образом, что

$$
\varphi_{1}(N+1)-q \varphi_{1}(N)=q^{-N},
$$

то

$$
\varphi_{2}(N+1)=q \varphi_{2}(N)
$$

следовательно,

$$
\varphi_{2}(N)=c^{\prime} q^{N}, \quad c^{\prime} \text { - константа. }
$$

Легко проверить, что $\varphi_{1}(N)$ может быть выбрана следующим образом:

$$
\begin{aligned}
& \varphi_{1}(N)=\frac{q^{N}-q^{-N}}{q-q^{-1}}, \quad q \neq-1, \\
& \varphi_{1}(N)=N e^{i \pi(N+1)}, \quad q=-1 .
\end{aligned}
$$

Из $(22),(24),(25)$ и $\left(25^{\prime}\right)$ получаем, что

$$
\begin{gathered}
a^{+} a=\frac{c q^{N}-q^{-N}}{q-q^{-1}} \equiv[N]_{c, q}, \quad c=1+c^{\prime}\left(q-q^{-1}\right), \quad q \neq-1, \\
a^{+} a=(N+c) e^{i \pi(N+1)} \equiv[N]_{c,-1}, \quad c=-c^{\prime}, \quad q=-1 .
\end{gathered}
$$

Таким образом, при фиксированном $c$-алгебра является одной из алгебр, описываемых формулой (3). Вместе с тем соотношение (1) не фиксирует однозначно связь между $a^{+} a$ и $N$. Ниже для краткости мы опускаем $q$ в $[N]_{c, q}$.

В случае $q$-алгебры формулы (17) и (18) имеют вид

$$
\begin{aligned}
\left\langle\psi_{\lambda+n}, \psi_{\lambda+n}\right\rangle & =[\lambda+n]_{c}[\lambda+n-1]_{c} \ldots[\lambda+1]_{c}\left\langle\psi_{\lambda}, \psi_{\lambda}\right\rangle, \\
\left\langle\psi_{\lambda-n}, \psi_{\lambda-n}\right\rangle & =[\lambda-n+1]_{c}[\lambda-n+2]_{c} \ldots[\lambda]_{c}\left\langle\psi_{\lambda}, \psi_{\lambda}\right\rangle .
\end{aligned}
$$


$\operatorname{Sp} N$ может быть ограничен сверху или снизу, только если $[\lambda+k]_{c}=0$ при некотором $k$, т.е. если

$$
\begin{array}{ll}
c \equiv c_{k}=q^{-2(\lambda+k)}, & q \neq-1, \\
c \equiv c_{k}=-(\lambda+k), & q=-1 .
\end{array}
$$

Согласно (27), $\left(27^{\prime}\right)$, если $q \in \mathbb{R}$, то спектр $N$ может быть или неограниченным, или ограниченным только снизу или сверху.

Легко показать, что т.к. $a^{+} a$ и $N$ - самосопряженные операторы, то $c \in \mathbb{R}$. Кроме того, если $q<0$, то $\lambda$ - целое число.

Действительно, если $q=-\tilde{q}, \tilde{q}>0$, то согласно (1)

$$
\left\langle\psi_{\lambda+k}, a a^{+}+\tilde{q} a^{+} a \psi_{\lambda+k}\right\rangle=\tilde{q}^{-(\lambda+k)} e^{i \pi(\lambda+k)} .
$$

Левая часть этого равенства действительна, следовательно, $\lambda \in \mathbf{Z}$. Если $q \in \mathbb{C}$, то $|q|=1, c=1$ (см. приложение).

Согласно теореме (см. $\left.\left(17^{\prime}\right),\left(18^{\prime}\right)\right)$ два представления $q$-алгебры (квази-)унитарноэквивалентны, если $\mathrm{Sp} N_{1}=\mathrm{Sp} N_{2}$ и $c_{1}=c_{2}$. Ниже мы не будем различать такие представления.

Рассмотрим теперь вопрос, в каких случаях, т.е. при каких $c$ и $q$, метрика является индефинитной, а при каких - положительно-определенной. Прежде всего заметим, что если $[\lambda+k]_{c}$ заданы, то согласно $\left(17^{\prime}\right),\left(18^{\prime}\right)$ изменение знака $\left\langle\psi_{\lambda+k}, \psi_{\lambda+k}\right\rangle$ при некотором $k=k_{0}$ приводит к изменению знака у всех $\left\langle\psi_{\lambda+k}, \psi_{\lambda+k}\right\rangle$. Такая замена соответствует квазиунитарному преобразованию, и, поскольку мы не различаем представления, связанные таким преобразованием, знак $\left\langle\psi_{\lambda+k_{0}}, \psi_{\lambda+k_{0}}\right\rangle$ можно выбрать произвольным образом.

Проанализирум зависимость знаков $\left\langle\psi_{\lambda+k}, \psi_{\lambda+k}\right\rangle$ от величины $c$.

Рассмотрим отдельно несколько случаев.

1а. $q>0, c>0$. Разобьем действительную ось на интервалы: $I_{k}(q) \equiv I_{k}, c \in I_{k_{0}}$, если

$$
\begin{array}{rlrl}
c_{k_{0}} & <c<c_{k_{0}-1}, & q>1, \\
c_{k_{0}-1}<c<c_{k_{0}}, & q<1,
\end{array}
$$

где $c_{k}=q^{-2(\lambda+k)}$.

Пусть $c \neq c_{k} \forall k$. Тогда если $q>1$ и $c \in I_{k_{0}}$, то согласно $\left(17^{\prime}\right),\left(18^{\prime}\right)[\lambda+k]_{c}>0$ при $k \geq k_{0}$ и $[\lambda+k]_{c}<0$ при $k<k_{0}$. Положим $\left\langle\psi_{\lambda+k_{0}}, \psi_{\lambda+k_{0}}\right\rangle>0$. Тогда из формул $\left(17^{\prime}\right)$, $\left(18^{\prime}\right)$ непосредственно следует, что

$$
\begin{aligned}
& \left\langle\psi_{\lambda+k}, \psi_{\lambda+k}\right\rangle>0, \quad k \geq k_{0}, \\
& \left\langle\psi_{\lambda+k}, \psi_{\lambda+k}\right\rangle=(-1)^{k_{0}-k}\left|\left\langle\psi_{\lambda+k}, \psi_{\lambda+k}\right\rangle\right|, \quad k<k_{0} .
\end{aligned}
$$

Если $q<1$ и с $\in I_{k_{0}}$, то, считая, как и раньше, $\left\langle\psi_{\lambda+k_{0}}, \psi_{\lambda+k_{0}}\right\rangle>0$, видим, что теперь

$$
\begin{array}{llrl}
\left\langle\psi_{\lambda+k}, \psi_{\lambda+k}\right\rangle & >0, & & k \leq k_{0}, \\
\left\langle\psi_{\lambda+k}, \psi_{\lambda+k}\right\rangle & =(-1)^{k-k_{0}}\left|\left\langle\psi_{\lambda+k}, \psi_{\lambda+k}\right\rangle\right|, & & k>k_{0} .
\end{array}
$$


Таким образом, когда $c>0$ и спектр $N$ неограничен, метрика всегда индефинитна.

Если $c=c_{k}$ (см. (27)), спектр $N$ ограничен сверху или снизу. При $q>1$ метрика положительна, если спектр ограничен снизу, и индефинитна, если - сверху; при $q<1$ наоборот.

1б. $q>0, c<0$. В этом случае, если $q>1$, то $[\lambda+k]_{c}<0 \forall k$; если $q<1$, то $[\lambda+k]_{c}>0 \forall k$. Таким образом, если $q>1$, то метрика индефинитна, причем, если $\psi_{\lambda+k}-$ положительный вектор, то $\psi_{\lambda+k \pm 1}-$ отрицательные векторы. Если $q<1$, то метрику всегда можно считать положительно-определенной.

2. $q<0$. Тогда формула (26) может быть переписана в виде

$$
a^{+} a=e^{i \pi(N+1)}[N]_{c, \tilde{q}}, \quad q=-\tilde{q} .
$$

Согласно $\left(26^{\prime}\right)$ и $\left(26^{\prime \prime}\right)$ при $q<0$ метрика всегда индефинитна. Тем же способом, что и раньше, легко определить знаки $\left\langle\psi_{\lambda+k}, \psi_{\lambda+k}\right\rangle$.

Из проведенного анализа следует, что, вообще говоря, при заданном $N$ вешественная константа $c$ не может быть произвольной. Действительно, пусть $a, a^{+}, N$ - некоторое представление $q$-алгебры, $a^{+} a=[N]_{c}$. Предположим, что существует другое представление $q$-алгебры: $a^{\prime}, a^{\prime+}, N, a^{++} a^{\prime}=[N]_{c^{\prime}}$. Докажем, что это возможно лишш тогда, когда

$$
\frac{[\lambda+k]_{c}}{[\lambda+k]_{c^{\prime}}}>0 \quad \forall k
$$

Действительно, в соответствии со сделанным предположением $a^{\prime+} \psi_{\lambda}=\psi_{\lambda+1}^{\prime}$ есть собственный вектор оператора $N$. Поскольку мы рассматриваем случай простого спектра оператора $N$, то $\psi_{\lambda+1}^{\prime} \sim \psi_{\lambda+1}$. Однако согласно (26), $\left(26^{\prime}\right)$

$$
\begin{aligned}
\left\langle\psi_{\lambda+1}, \psi_{\lambda+1}\right\rangle & =[\lambda+1]_{c}\left\langle\psi_{\lambda}, \psi_{\lambda}\right\rangle, \\
\left\langle\psi_{\lambda+1}^{\prime}, \psi_{\lambda+1}^{\prime}\right\rangle & =[\lambda+1]_{c^{\prime}}\left\langle\psi_{\lambda}, \psi_{\lambda}\right\rangle .
\end{aligned}
$$

Условие $\psi_{\lambda+1}^{\prime} \sim \psi_{\lambda+1}$ означает, что векторы $\psi_{\lambda+1}^{\prime}$ и $\psi_{\lambda+1}$ либо оба положительны, либо оба отрицательны. Требуемое условие доказано для $k=1$. Для произвольного $k$ оно вытекает из аналогичных соотношений. Если $q>0, c>0$, то из формул (29) $\left(30^{\prime}\right)$ следует, что условие (31) может быть выполнено, только если $c$ и $c^{\prime}$ принадлежат одному и тому же интервалу $I_{k}$. Кроме того, легко увидеть, что соотношения $(29)-\left(30^{\prime}\right)$ фиксируют тот интервал $I_{k}$, которому может принадлежать $c$.

\section{5. НЕУНИТАРНЫЕ ПРЕДСТАВЛЕНИЯ, ОТНОСИТЕЛЬНО КОТОРЫХ $q$-АЛГЕБРА ИНВАРИАНТНА, СЛУЧАЙ $q>0$}

Пусть операторы $a, a^{+}$и $N$ принадлежат $q$-алгебре $(1), q>0$. Тогда в рассматриваемом пространстве существует набор других представлений этой алгебры. Новые представления могут быть получены с помощью следующих преобразований:

1. "Скейлинговые" преобразования. Если

$$
a^{\prime}=q^{\nu / 2} a, \quad\left(a^{\prime}\right)^{+}=q^{\nu / 2} a^{+}, \quad N^{\prime}=N-\nu, \quad \nu \in \mathbb{R}
$$

то простое вычисление показывает, что $a^{\prime},\left(a^{\prime}\right)^{+}$и $N^{\prime}$ принадлежат $q$-алгебре так же, как $a, a^{+}$и $N$. Согласно $(26)$

$$
c^{\prime}=q^{2 \nu} c
$$


2. Преобразования, оставляюшие $N$ инвариантным. Пусть

$$
a^{\prime}=\varphi(N) a, \quad\left(a^{\prime}\right)^{+}=a^{+} \varphi(N) .
$$

Тогда $a^{\prime},\left(a^{\prime}\right)^{+}$и $N$ принадлежат $q$-алгебре, если (см. (26))

$$
\left(a^{\prime}\right)^{+} a^{\prime}=[N]_{c^{\prime}}, \quad a^{\prime}\left(a^{\prime}\right)^{+}=[N+1]_{c^{\prime}} .
$$

Следовательно, согласно (34)

$$
\begin{gathered}
{[N+1]_{c^{\prime}}=\varphi(N) a a^{+} \varphi(N)=\varphi^{2}(N)[N+1]_{c},} \\
\varphi(N)=\left(\frac{[N+1]_{c^{\prime}}}{[N+1]_{c}}\right)^{1 / 2} .
\end{gathered}
$$

Из формул (34) следует, что $\varphi(N)=\varphi^{+}(N)$. Поскольку

$$
\varphi(N) \varphi^{+}(N) \psi_{\lambda+k}=\varphi(\lambda+k) \varphi^{*}(\lambda+k) \psi_{\lambda+k},
$$

то формула (35) справедлива, если выполнено условие (31). Согласно проведенному в конце предыдушего раздела анализу, это условие выполняется при произвольном $c<0$. При $c>0$ условие (31) будет выполнено, когда $c$ и $c^{\prime}$ принадлежат одному и тому же $I_{k}$.

Если в $J$ сушествует некоторое представление $q$-алгебры, то, используя преобразования (32) и (34), мы можем построить любое представление с тем же знаком $c$ и тем же видом спектра $N$ (неограниченного или ограниченного сверху (или снизу)).

Действительно, если в заданном представлении $a, a^{+}, N$ соответствующее $c>0$ и $c \neq c_{k}$ (спектр $N$ неограничен), то, используя преобразование (32), мы всегда можем прийти к представлению $a^{\prime},\left(a^{\prime}\right)^{+}, N^{\prime}$ с произвольным неограниченным спектром $N^{\prime}$ и $c^{\prime}$, принадлежашим любому интервалу $I_{k}$. Затем с помошью преобразования (34) мы можем связать это представление с представлением $a^{\prime \prime},\left(a^{\prime \prime}\right)^{+}, N^{\prime \prime}=N^{\prime}$, где $c^{\prime \prime}$ - любая константа, принадлежашая интервалу $I_{k}$. Если $c=c_{k}$ и $\operatorname{Sp} N$ ограничен сверху (снизу), то с помошью преобразования (32) мы можем прийти к любому представлению $a^{\prime},\left(a^{\prime}\right)^{+}$, $N^{\prime}$, где $N^{\prime}$ ограничен сверху (снизу). Аналогично мы можем связать любое заданное представление $a, a^{+}, N$ при $c<0$ с произвольным представлением $a^{\prime},\left(a^{\prime}\right)^{+}, N^{\prime}$, если только $c^{\prime}<0$.

Таким образом, когда $q>0$, два произвольных представления $q$-алгебры связаны комбинацией унитарного и неунитарного преобразований, описанных вьше, если только знаки соответствуюших констант и типы спектров соответствуюших операторов совпадают.

\section{6. НЕУНИТАРНЫЕ ПРЕОБРАЗОВАНИЯ, ОТНОСИТЕЛЬНО КОТОРЫХ $q$-АЛГЕБРА ИНВАРИАНТНА, СЛУЧАЙ $q<0$}

При $q=-\tilde{q}, \quad \tilde{q}>0$ мы рассмотрим только случай $\tilde{q} \neq 1$, т.к. случай $\tilde{q}=1$ может быть исследован аналогично.

Согласно $\left(26^{\prime \prime}\right)$ условие (27) принимает вид

$$
c_{k}=\tilde{q}^{-2(\lambda+k)} .
$$


Напомним, что $\operatorname{Sp} N$ состоит из целых чисел (см. раздел 4).

Преобразование (32) справедливо теперь, только если $\nu=2 l, l \in \mathbf{Z}$, следовательно,

$$
N^{\prime}=N-2 l, \quad c^{\prime}=q^{4 l} c .
$$

Преобразование (34) остается неизменным. Как и раньше, оно возможно, только если $c$ и $c^{\prime}$ принадлежат одному и тому же интервалу $I_{k}$ (см. $\left.(28),\left(28^{\prime}\right)\right)$. Однако теперь аналогичные условия формулируются для $\tilde{q}$.

Рассмотрим отдельно случаи $c>0$ и $c<0$.

1a. $c>0, c \neq c_{k}$. Начиная с некоторого представления $q$-алгебры мы можем построить другие, используя преобразования $\left(32^{\prime}\right)$ и (34). Разница со случаем, когда $q>0$, состоит в том, что теперь мы можем связать два представления $a, a^{+}, N, c$ и $a^{\prime},\left(a^{\prime}\right)^{+}$, $N^{\prime}, c^{\prime}$, только если $c \in I_{2 k}$ и $c^{\prime} \in I_{2 l}$ или $c \in I_{2 k+1}$ и $c^{\prime} \in I_{2 k+1}$.

1б. $c>0, c=c_{k}$. В этом случае, если $c \in I_{2 k}\left(I_{2 k+1}\right)$ и $c^{\prime} \in I_{2 l}\left(I_{2 l+1}\right)$, соответствуюшие представления могут быть связаны преобразованием $\left(32^{\prime}\right)$.

2. $c<0$. При этом знак $[l]_{c}$ не зависит от $c$. Если $\tilde{q}>1$, то $[l]_{c}>0$. Если $\tilde{q}<1$, то $[l]_{c}<0$. Заданное представление $a, a^{+}, N, c$ может быть связано с любым представлением $a^{\prime},\left(a^{\prime}\right)^{+}, N, c^{\prime}$ преобразованием (34), если только $c^{\prime}<0$.

Нам осталось рассмотреть свойства $q$-алгебры при $q \in \mathbb{C}$. В приложении показано, что тогда $q=\exp (i \varphi), \varphi \in \mathbb{R}, c=1$. Очевидно, что здесь преобразование (32) тривиально. Следовательно, мы можем рассмотреть только вид спектра $N$. Напомним, что в гильбертовом пространстве этот спектр всегда конечен и только выделенные значения $\varphi$ возможны, точнее, $q=\sqrt[n]{-1}$. В пространстве с индефинитной метрикой все значения $\varphi$ возможны и спектр $N$ может быть ограничен, полуограничен или неограничен. Используя формулы $\left(17^{\prime}\right)$ и $\left(18^{\prime}\right)$, легко получить связь между $\varphi$ и видом спектра оператора $N$ (см. приложение).

\section{7. СВЯЗЬ МЕЖДУ ПРЕДСТАВЛЕНИЯМИ q-АЛГЕБРЫ ПРИ РАЗЛИЧНЫХ $q$}

Покажем, что сушествование в пространстве $J$ представления $q$-алгебры влечет за собой сушествование представлений аналогичной алгебры, но отвечающей другому значению $q: q \rightarrow q^{\prime}$.

Как обычно, рассмотрим отдельно случаи $q>0$ и $q<0$.

1. $q>0$. Пусть спектр $N$ неограничен, т.е. $c(q) \neq c_{k}(q)$ и $c(q)>0$. Докажем, что тогда существуют представления $q^{\prime}$-алгебры при любом $q^{\prime}>0$ и любом $c\left(q^{\prime}\right) \neq c_{k}\left(q^{\prime}\right)$, если $c\left(q^{\prime}\right)>0$.

Сначала найдем преобразование, переводяшее $q$-алгебру в $q^{\prime}$-алгебру в том случае, когда $q>1(q<1), q^{\prime}>1\left(q^{\prime}<1\right)$.

Пусть $c(q) \in I_{k}(q)$. Выберем $c\left(q^{\prime}\right)$ таким образом, чтобы $c\left(q^{\prime}\right) \in I_{k}\left(q^{\prime}\right)$. Согласно результатам раздела 5 знаки величин $[\lambda+l]_{c, q}$ и $[\lambda+l]_{c^{\prime}, q^{\prime}}$ совпадают при всех $l$, если $q>1, q^{\prime}>1$ или $q<1, q^{\prime}<1$. Поэтому оператор $\varphi_{q, q^{\prime}}(N)=[N+1]_{c, q} /[N+1]_{c^{\prime}, q^{\prime}}$ хорошо определен. Проведя те же рассуждения, что и при выводе формулы (34), получим, что операторы $N$ и

$$
\begin{aligned}
a\left(q^{\prime}\right) & =\varphi_{q, q^{\prime}}(N) a(q), \\
a^{+}\left(q^{\prime}\right) & =a^{+}(q) \varphi_{q, q^{\prime}}(N)
\end{aligned}
$$


удовлетворяют $q^{\prime}$-алгебре. Далее, применяя к $q^{\prime}$-алгебре преобразования $(32),(34)$, получим произвольное представление этой алгебры при $c\left(q^{\prime}\right)>0, c\left(q^{\prime}\right) \neq c_{k}\left(q^{\prime}\right)$. Осталось установить связь между операторами $q$ - и $q^{\prime}$-алгебр, если $q>1, q^{\prime}<1$ или наоборот.

Согласно (32) произвольное представление, для которого $c\left(q^{\prime}\right)>0$, можно связать с представлением, в котором $c(q)=1$. Однако, если $c(q)=1$, то согласно $(26)$ $[N]_{q}=[N]_{q^{-1}}$ и соответствующие операторы $a(q), a^{+}(q), N$ удовлетворяют не только $q$-алгебре, но и $q^{-1}$-алгебре. Далее к $q^{-1}$-алгебре можно применить рассуждения, проведенные для $q$-алгебры. Сделанное утверждение полностью доказано.

Если $c(q)=c_{k}(q)$, то с помощью преобразования $(32)$ перейдем к представлению, в котором $c=1$. В этом случае есть две возможности: $\operatorname{Sp} N=\mathbb{N}$ и $\operatorname{Sp} N=\mathbf{Z}_{-}$. Согласно (26) в первом случае все $[l]_{1, q}>0$, во втором все $[l]_{1, q}<0$. Поскольку эти неравенства не зависят от $q$, то и в первом, и во втором случаях существует преобразование, аналогичное (36), если $c^{\prime} \in c_{l}\left(q^{\prime}\right)$.

Осталось рассмотреть случай $c(q)<0$. В этом случае, если $q<1$, то все $[\lambda+l]_{q, c}>0$; если $q>1$, то все $[\lambda+l]_{q, c}<0$. Преобразование $(36)$ сушествует, если $q>1$ и $q^{\prime}>1$ или $q<1$ и $q^{\prime}<1$, при этом $c\left(q^{\prime}\right)<0$.

2. $q<0$. Ограничимся рассмотрением случая $q \neq-1$. Пусть $c \neq c_{k}, c>0$. Согласно результатам раздела 5 представления распадаются на два класса: $c \in I_{2 k}$ и $c \in I_{2 k+1}$. Преобразования $(32),(34)$ сушествуют только для представлений, принадлежаших одному и тому же классу. Соответственно из существования $q$-алгебры определенного класса $\left(c \in I_{2 k}\right.$ или $\left.c \in I_{2 k+1}\right)$ следует сушествование $q^{\prime}$-алгебры того же класса. При этом $q^{\prime}>1$, если $q>1$, или $q^{\prime}<1$, если $q<1$.

Если $c \in c_{2 k}$ или $c \in c_{2 k+1}$, то мы приходим к тем же результатам. В случае, если $c \in$ $c_{2 k}$, с помошью преобразования $\left(32^{\prime}\right)$ можно получить представление с $c=1$, которое, как и при $q>0$, является одновременно представлением $q$ - и $q^{-1}$-алгебры.

\section{8. ОБЩЕЕ ПРЕДСТАВЛЕНИЕ РАССМАТРИВАЕМЫХ АЛГЕБР В СЛУЧАЕ ДИСКРЕТНОГО СПЕКТРА ОПЕРАТОРА $N$}

В этом разделе мы покажем, что если оператор $N$ имеет дискретный спектр и каждому собственному значению оператора $N$ соответствует неприводимое представление алгебры, заданной соотношениями (2) и (3), построенное описанным в разделе 3 способом, то произвольное представление рассматриваемых алгебр есть прямая сумма представлений, описанных в разделе 3 .

Действительно, если $\psi_{\lambda_{1}}^{1}-$ собственный вектор оператора $N$, то неприводимое представление рассматриваемой алгебры можно построить так же, как в разделе 3.

Обозначим соответствующее пространство через $J_{1}$. Если $J \neq J_{1}$, то рассмотрим пространство $J-J_{1}$, в этом пространстве выделим собственный вектор $\psi_{\lambda_{1}}^{2}$ ( или $\psi_{\lambda_{2}}^{1}$, если собственному значению $\lambda_{1}$ соответствует один собственный вектор) и построим неприводимое представление, соответствуюшее этому вектору. Продолжая этот процесс, получим $J \supset J_{1}+J_{2}+\cdots$. Докажем, что в действительности

$$
J=J_{1}+J_{2}+\cdots
$$

Для этого заметим, что множество пространств $J_{i}$ может быть частично упорядочено естественным образом по включению: $J_{i} \subset J^{i}$, где $J^{i}=\sum_{k=1}^{i} J_{k}$. Согласно лемме 
Цорна такое множество имеет максимальный элемент $J^{\max }$. Однако если $J \neq J^{\max }$, то в пространстве $J-J^{\text {max }}$ у оператора $N$ должен быть собственный вектор и, следовательно, обычным образом можно построить пространство $\tilde{J}$, соответствуюшее этому вектору. Пространство $J^{\max }+\tilde{J}$, очевидно, принадлежит системе пространств $J_{i}$, и включение $J^{\max } \subset J^{\max }+\tilde{J}$ находится в противоречии с определением $J^{\text {max }}$. Следовательно, $J^{\max }=J$ и равенство (37) доказано. Полученное разложение фактически является частным случаем разложения общего представления некоторой алгебры на ее циклические представления [16, с. 286].

\section{ПРИЛОЖКЕНИЕ}

Как известно, в случае положительной метрики, если $q \in \mathbb{C}$, то $\operatorname{Sp} N$ конечен, а соответствуюшие представления сушествуют только при выделенных значениях $q$, а именно, $q=\sqrt[n]{-1}$

В случае индефинитной метрики набор различных представлений намного шире: $\varphi$ может быть любым $\left(q=e^{i \varphi}\right)$, и спектр $N$ может быть любого типа, т.е. неограниченным, полуограниченным снизу (сверху) или ограниченным.

Прежде всего докажем, что для индефинитной метрики, так же как и для положительной метрики, $|q|=1$ (согласно (27)), в противном случае $\operatorname{Sp} N$ или неограничен или полуограничен. Предполагая, что спектр $N$ неограничен сверху, рассмотрим (26) при $n \rightarrow \infty$. Простые выкладки показывают, что условие $\left(a^{+} a\right)^{+}=a^{+} a$ не может быть выполнено, если $|q| \neq 1$. Случай неограниченного снизу спектра $N$ рассматривается аналогично.

Для вывода равенства $c=1$ достаточно записать $c$ в виде $c=1+c^{\prime}\left(q-q^{-1}\right)$ и заметить, что в данном случае условие $N=N^{+}$эквивалентно условию самосопряженности оператора $c^{\prime} e^{i N \varphi}$, которое выполнено, если только $c^{\prime}=0$.

Итак, доказано, что $q=e^{i \varphi}, 0<\varphi<2 \pi$. Ниже мы считаем, что $0<\varphi<\pi$, поскольку случай $\pi<\varphi<2 \pi$ рассматривается аналогично. Для конкретности пусть $-1<\lambda<1$ (см. (4)). Общий случай легко может быть проанализирован тем же способом. Заметим, что если $\lambda \neq 0$, то метрика всегда индефинитна. Действительно, если $\lambda>0$, то $[\lambda]>0$, а $[\lambda-1]<0$; если $\lambda<0$, то $[\lambda]<0,[\lambda+1]>0$.

Элементарные выкладки показывают, что спектр $N$ ограничен сверху, если

$$
\varphi=\frac{n_{1} \pi}{\lambda+m_{1}}, \quad n_{1}, m_{1} \in \mathbf{Z}^{+},
$$

снизу, если

$$
\varphi=\frac{n_{2} \pi}{m_{2}-\lambda}, \quad n_{2}, m_{2} \in \mathbf{Z}^{+},
$$

и содержит конечное число собственных векторов, если равенства (П.1) и (П.2) справедливы одновременно. В этом случае

$$
\lambda=\frac{n_{1} m_{2}-m_{1} n_{2}}{n_{1}+n_{2}} .
$$

В остальных случаях спектр $N$ неограничен. 


\section{Список литературы}

[1] L. D. Faddeev. Integrable models in $(1+1)$-dimensional quantum field theory. In: Les Houches Lectures. Amsterdam: Elsevier, 1982. P. 563.

[2] P.P. Kulish, E. K. Sklyanin. In: Lectures Notes in Physics. V. 151. Berlin, Heidelberg, New York: Springer-Verlag, 1981. P. 61.

[3] M. Jimbo. Int. J. Mod. Phys. 1989. V. 4. P. 3759

[4] M. Chaichian, P. P. Kulish. Phys. Lett. B. 1990. V. 234. P. 72.

[5] P. P. Kulish, E. V. Damaskinsky. J. Phys. A: Math. Gen. 1990. V. 23. P. L415.

[6] П. П. Кулиш. ТМФ. 1991. Т. 86. С. 157.

[7] A. Kempf. J. Math. Phys. 1993. V. 34. P. 969.

[8] A. A. Andrianov, F. Cannata, J.-P. Dedonder, M. V. Ioffe. Phys. Lett. A. 1996. V. 217. P. 7.

[9] M. Chaichian, M. N. Mnatsakanova, Yu.S. Vernov. In: Proc. of the XV Workshop "Problems on High Energy Physics and Field Theory" (Protvino, 1992). Protvino: IHEP, 1995. P. 126; M. N. Mnatsakanova, Yu.S. Vernov. In: Proc. of the XVIII Workshop "Problems on High Energy Physics and Field Theory". Protvino: IHEP, 1996. P. 337.

[10] M. Chaichian, M. N. Mnatsakanova, Yu.S. Vernov. J. Phys. A: Math. Gen. 1994. V. 27. P. 2053.

[11] I. Ya. Aref'eva. Quantum Group Gauge Fields. Preprint CERN-TH.6207/91. Geneva: CERN, 1991.

[12] F. Strocchi, A.S. Wightman. J. Math. Phys. 1974. V. 15. P. 2198.

[13] F. Strocchi. Selected Topics on the General Properties of Quantum Field Theory. Singapore: World Scientific, 1993.

[14] G. Morchio, F. Strocchi. Ann. Inst. Henri Poincaré. 1980. V. 33. P. 251.

[15] C. R. Putnam. Commutation Properties of Hilbert Space Operators and Related Topics. Berlin, Heidelberg, New York: Springer-Verlag, 1967.

[16] М. А. Наймарк. Нормированные кольца. М.: Наука, 1968.

[17] J. Bognar. Indefinite Inner-Product Spaces. Berlin, Heidelberg, New York: Springer-Verlag, 1974.

[18] Т. Я. Азизов, И. С. Иохвидов. Основы теории линейных операторов в пространствах с индефинитной метрикой. М.: Наука, 1986.

[19] M. Mnatsakanova, G. Morchio, F. Strocchi, Yu. Vernov. Irreducible representations of the Heisenberg algebra in Krein spaces. Preprint IFUP-TH-95. Pisa: INFN, 1995.

Поступила в редакцию 26.III.1997 г., после доработки 19.VI.1997 г.

\section{Yu.S. Vernov, M. N. Mnatsakanova \\ ALGEBRA OF $q$-DEFORMED COMMUTATORS IN AN INDEFINITE METRIC SPACE}

Representations and invariant properties of $q$-deformed commutator algebra and its generalization are investigated in an indefinite metric space. 